\title{
Cancer of the ampulla of Vater: analysis of the whole genome sequence exposes a potential therapeutic vulnerability
}

\author{
Michael J Demeure ${ }^{1,2^{*}}$, David W Craig ${ }^{3}$, Shripad Sinari ${ }^{3}$, Tracy M Moses ${ }^{1}$, Alexis Christoforides ${ }^{3}$, Jennifer Dinh', \\ Tyler Izatt ${ }^{3}$, Jessica Aldrich ${ }^{3}$, Ardis Decker ${ }^{4}$, Angela Baker ${ }^{1}$, Irene Cherni ${ }^{1}$, April Watanabe ${ }^{1}$, Lawrence Koep ${ }^{5}$, \\ Douglas Lake ${ }^{6}$, Galen Hostetter ${ }^{1}$, Jeffrey M Trent ${ }^{1}$, Daniel D Von Hoff ${ }^{2,4}$ and John D Carpten ${ }^{1}$
}

\begin{abstract}
Background: Recent advances in the treatment of cancer have focused on targeting genomic aberrations with selective therapeutic agents. In rare tumors, where large-scale clinical trials are daunting, this targeted genomic approach offers a new perspective and hope for improved treatments. Cancers of the ampulla of Vater are rare tumors that comprise only about $0.2 \%$ of gastrointestinal cancers. Consequently, they are often treated as either distal common bile duct or pancreatic cancers.

Methods: We analyzed DNA from a resected cancer of the ampulla of Vater and whole blood DNA from a 63 year-old man who underwent a pancreaticoduodenectomy by whole genome sequencing, achieving $37 \times$ and 40x coverage, respectively. We determined somatic mutations and structural alterations.

Results: We identified relevant aberrations, including deleterious mutations of KRAS and SMAD4 as well as a homozygous focal deletion of the PTEN tumor suppressor gene. These findings suggest that these tumors have a distinct oncogenesis from either common bile duct cancer or pancreatic cancer. Furthermore, this combination of genomic aberrations suggests a therapeutic context for dual mTOR/PI3K inhibition.
\end{abstract}

Conclusions: Whole genome sequencing can elucidate an oncogenic context and expose potential therapeutic vulnerabilities in rare cancers.

\section{Background}

Advances in treatments for cancer have generally come incrementally because novel treatments are subjected to large prospective randomized clinical trials. In these studies, several hundred patients are randomized to one treatment arm or another and the treatment associated with the best outcome is advanced. This method has worked well for relatively common cancers, including breast and colon cancers. This approach, however, falls short when one is faced with rare cancers such that prospective trials involving large numbers of patients are difficult or impossible to conduct. In these cases, oncologists may choose chemotherapy regimens because the rare

\footnotetext{
* Correspondence: mdemeure@tgen.org

'Integrated Cancer Genomics Division, Translational Genomics Research Institute, 445 N. Fifth Ave, Phoenix, AZ 85004, USA

Full list of author information is available at the end of the article
}

tumor is thought to be similar to a more common cancer for which an accepted standard treatment exists. Such is the case with cancers of the ampulla of Vater. These cancers account for only $0.2 \%$ of gastrointestinal cancers and approximately $7 \%$ of periampullary tumors. Periampullary tumors arise from either pancreatic ductal epithelium, the distal common bile duct, the duodenal mucosa, or the ampulla of Vater. When resectable, ampullary cancers are treated like pancreatic cancers with a pancreaticoduodenectomy. When they present at an advanced metastatic stage, there is little information guiding choices for chemotherapy regimens. Although they represent a minority in such trials, patients with ampullary cancers are often included in clinical trials of patients with biliary tract cancers, so these patients are often treated with gemcitabine and cisplatin [1].

\section{Biomed Central}


Genomic technologies have resulted in some limited but remarkable advances in cancer treatment. Prior to the discovery of the Philadelphia chromosome and the identification of the $B C R / A B L$ fusion protein leading to the development of imatinib, chronic myelogenous leukemia, a relatively rare form of the disease, was nearly uniformly fatal. Treatment was a bone marrow transplant with its attendant high risks of both morbidity and death. Treatment with imatinib, a tyrosine kinase inhibitor, can induce remission in approximately $87 \%$ of patients with greatly reduced risks of complications [2]. Imatinib was subsequently also found to be remarkably effective against gastrointestinal stromal tumors [3]. Other targeted drugs that have recently been shown to have efficacy in the setting of an indentified genomic aberration include vismodegib in advanced basal cell skin cancers harboring mutations in $P T C H 1$, and vemurafenib in patients with advanced melanoma exhibiting a V600E mutation in the $B R A F$ (v-raf murine sarcoma viral oncogene homolog B1) gene product $[4,5]$.

The rapid advancement of genomic technologies offers the possibility to tailor chemotherapy based on an indepth analysis of a limited number of tumor samples. The advent of next generation sequencing technologies has now paved the way for near complete interrogation of tumor genomes, providing the first opportunity for efficient global genomic tumor profiling at the point mutation, copy number, and breakpoint dimensions of the cancer genome. At a time in which there is an increasing array of chemotherapy drugs targeting aberrant molecular pathways, individualized genomic analysis to aid treatment decisions is quickly becoming feasible. Such an approach seems particularly well suited to the treatment of rare cancers for which there is a paucity of other clinical data to guide therapy. To demonstrate the potential clinical utility of individualized genomic analysis in patients with rare cancers, we applied whole genome sequencing to the tumor of a 63-year-old man with a resected cancer of the ampulla of Vater and identified therapeutic targets distinct from what would have been targeted based on existing literature.

\section{Materials and methods Samples}

Written informed consent was obtained and the patient samples were collected for research purposes at Banner Good Samaritan Medical Center, Phoenix, Arizona. The study was approved by the Western Institutional Review Board (WIRB) and was conducted in accordance with the 1996 Declaration of Helsinki. This was a study entitled, 'Pancreas Cancer Biospecimens Repository' (WIRB ${ }^{\circledR}$ Protocol \#20040832). Informed consent was obtained from the patient with cancer of the ampulla of Vater, including written consent for collection of the tissue and whole blood samples as well as clinical information and for genetic analysis of the specimens. The samples were then anonymized and assigned a unique identifier. Samples included fresh frozen tumor tissue collected within 20 minutes after surgical resection. Whole blood was obtained before the start of the operation at the time of induction of anesthesia. Histopathological analysis of the frozen specimen was quality assessed and determined to contain approximately $60 \%$ tumor cellularity. DNA and RNA were extracted from frozen tissue and whole blood using the Qiagen All Prep kit (Germantown, MD, USA) using the manufacturer's recommendations.

\section{Next generation sequencing}

To facilitate whole genome next generation sequencing, we utilized the Life Technologies SOLiD ${ }^{\mathrm{TM}}$ (version 3) technology with mate-pair chemistry using the manufacturer's recommendations (Carlsbad, CA, USA). Briefly, 20 $\mu \mathrm{g}$ of genomic DNA is mechanically sheared to an average fragment size of $1.5 \mathrm{~kb}$ using the HydroShear. These sizeselected fragments are then end repaired and circularized around a long mate-pair adaptor by nicked ligation. Nick translation is then used to displace the nick roughly $70 \mathrm{bp}$ from either side of the internal adaptor. A nuclease reaction linearizes these fragments. SOLiD ${ }^{\mathrm{TM}}$ sequencingspecific sequencing adaptors are then ligated to the ends of these fragments. We prepared two independent $1.5 \mathrm{~kb}$ mate-pair libraries from the patient's constitutional (germline) DNA, and two independent mate-pair libraries from the patient's tumor DNA. Following PCR amplification, these mate-pair libraries are then used as templates in emulsion $\mathrm{PCR}$ reactions using $\mathrm{SOLiD}^{\mathrm{TM}}$ proprietary sequencing beads to generate clonal single molecule templated beads. Subsequently, an average of 500,000 templated beads are enriched and deposited onto SOLiD ${ }^{\mathrm{TM}}$ flowcells for massive ligation-based sequencing to generate $50 \mathrm{bp} \times 50 \mathrm{bp}$ mate-pair sequences per bead. For this germline/tumor pair, we sequenced an average of one billion beads per library, thus generating two billion matepair reads for germline and two billion mate-pair reads for tumor.

\section{Next generation sequencing data processing}

Raw next generation sequencing data in the form of csfasta and qual files are used to align $50 \mathrm{bp} \times 50 \mathrm{bp}$ paired end reads from either the patient germline genome sequence or tumor genome sequence to the reference human genome (NCBI build 36, hg18). For alignment, we utilized the Life Technologies BioScope version 1.3 software suite, which is based upon a seed-and-extend algorithm [6]. Compressed binary sequence alignment/map (BAM) formatted output files for germline and tumor genome alignments are generated and PCR duplicates are subsequently removed using the Picard Tools. 


\section{Next generation sequencing data analysis Somatic single nucleotide variants}

We employed two different algorithms. The first algorithm (SolSNP) [7] detects a SNP variant by comparing two discrete distributions. It compares the distance of the discrete sampled distribution of the base-pair pileup on each strand to the expected distributions (according to ploidy), and determines the genotype call. This is done using a Kolmogorov-Smirnov-like distance measure based on both the base (that is, reference or alternative base) as well as the confidence in the base called (that is, the quality score of each base in the pileup). If the genome is haploid, two expected pileups are created at each position: one consisting of only the reference base (a 'homozygous-reference' pileup) and another consisting of only the alternative base (a 'homozygous non-reference' pileup). The confidence of each pileup position is kept the same. The expected pileup that has the minimal Kolmogorov-Smirnov distance to the sampled pileup is considered to be the genotype of the locus on the strand. In diploid genomes, SolSNP also considers a pileup half of which is made up of the reference bases and the other half made of alternative bases (a heterozygous pileup). A locus on the chromosome is called a SNP if a variant genotype (either 'homozygous non-reference' or heterozygous) is detected on both strands. SolSNP can restrict its calls to loci where the genotype calls on both strands are identical. This is achieved by passing the 'Genotype Consensus' value to the parameter 'STRAND_MODE'. In this mode, the tool is able to produce genotype calls as well as variants. The second algorithm (Mutation Walker) calculates a test of proportions for the tumor/normal set to construct a test-statistic for reads in the forward direction and the reverse detection separately. The minimum of these two comparisons is used as the reported test-statistic, ensuring evidence is found in both the normal and reverse detection. Sites with evidence in the normal are filtered from the final report so as to reduce false positives arising from under-sampled polymorphic germline events. Calls common to both the algorithms were considered for further examination. To reduce the false negative rate, two sets of common calls were made. One was made with a strict and the other with a lenient set of parameters for both the algorithms. Both the sets were visually examined for false positives, which were then filtered to get a final list of true single nucleotide variants.

\section{Indel detection}

For detecting somatic indels we employed a two-step strategy. In the first step, we removed reads from the tumor sample BAM whose insert size lay outside the interval $(500,5000)$ for SOLiD ${ }^{\mathrm{TM}}$. Genome Analysis Toolkit [8] was then used to generate a list of potential small indels from this BAM. A customized perl script, which used the
Bio-SamTools library from BioPerl [9], then took these indel positions and for each of the indels looked at the region in the germline sample consisting of five bases upstream of the start and five bases downstream of the end of the indel. An indel was determined to be somatic only if there was no indel detected in the region under consideration.

\section{Structural variants}

Structural variants were analyzed by comparing two sources of information: relative normal/tumor read-level coverage and anomalously mapping read pairs. Assessing structural variants by read-level coverage is termed copy-number analysis since it is parallel in concept to microarrays. In copy number analysis, gains and losses were determined by calculating the $\log 2$ difference in normalized coverage between tumor and germline. Briefly, we investigated regions in $100 \mathrm{bp}$ windows where the coverage in the germline was between 0.1 and 10 of the mode coverage in order to remove regions with high degrees of repeat sequence (for example, centromes or difficult to sequence regions. Normalized coverage was determined by the $\log 2$ coverage within a $100 \mathrm{bp}$ bin over the overall modal coverage. We then reported the difference between the germline and tumor normalized coverage by a sliding window of size $2 \mathrm{~kb}$. Deleted and amplified regions were flagged by a departure of greater than 0.75 from baseline. Moderate deletions were identified by a similar method utilizing sequence coverage rather than clonal coverage for consensus coding sequence exons only.

In anomalous read-pair analysis, we used perl scripts to detect enrichment of anomalously mapping read-pairs. These would be read-pairs that deviate from the expected mate-pair orientation of both reads occurring in the same direction or read-pairs that are outside the expected $1.5 \mathrm{~kb}$ insert size. A series of customized perl scripts were employed in the detection of translocation. These scripts used SAMtools [10] internally to access the BAM files. The analysis itself was made up of two steps. The first was the detection of a potential translocation in both tumor and germline samples. The second was comparison of a potential translocation in tumor to those detected in the germline sample to weed out potential false positives for statistical identification of outliers. The genome was analyzed by a walker with step size equivalent to the insert size where the number of anomalous reads was counted, that is, those reads whose mates align on a different chromosome. For each window we chose the highest hit to be the chromosome to which mates of most of the discordant reads mapped. We compared the ratios of discordant reads to the total aligned reads across all the windows to detect potential outliers. Outlier detection was done under the assumption that the normal distribution of the proportion of hit discordant reads in $2 \mathrm{~kb}$ windows aggregated 
across the chromosome will follow a normal distribution. We then computed the mean of the distributions and chose a cutoff of 3 standard deviations. The window with a proportion of hit discordant reads higher than this cutoff contained the region of potential translocation. The actual region of translocation is then determined by the span of the hit discordant reads in the window. For somatic translocations, the germline and the tumor sample are called separately and regions of overlap are eliminated. The output is a general feature format (gff) file of paired lines where the source tag indicates which two genomic regions show potential translocations. These regions were further inspected to reduce false positives and arrive at the more confident list. Additional details related to the methods for detection of somatic translocations and intrachromosomal rearrangements are included in Additional file 1.

\section{Validation of next generation sequencing findings}

Briefly, ten single nucleotide variants and one local deletion were selected at random for chain termination sequencing (Sanger method). Validation was conducted using tumor DNA. Specific genomic primer pairs (Additional file 2) were designed to anneal in flanking single nucleotide variant regions and approximately 150 to $500 \mathrm{bp}$ fragments to be amplified in 25-cycle PCR. Some primers carried M13 sequences on the 5 ' end as a back up for sequencing runs. Reaction products were column purified using a QIAquick PCR Purification kit (Qiagen) and submitted to the Arizona State University sequencing facility. Electropherograms were then manually examined for the presence of mutations/deletions in both orientations (Additional file 3).

Genomic quantitative PCR was performed to validate homozygous PTEN (phosphatase and tensin homolog) deletion (Additional file 4). In addition to the PTEN locus, genes located in adjacent regions of hemizygous deletion ( $R G R$ (retinal G protein coupled receptor) and HHEX (hematopoietically expressed homeobox)) were also measured. BICC1 (bicaudal C homolog 1 (Drosophila)) and TRUB1 (TruB pseudouridine (psi) synthase homolog 1), located in unaffected regions of chromosome 10, were used as internal controls. Quantitative PCR reactions were set up in a 384-well plate in triplicate with $3 \mathrm{ng}$ of genomic DNA input per reaction. Amplifications were performed using a LightCycler480 instrument and SYBRGreen I Master Mix (Roche). Melting curves were examined for the presence of a single peak and $\mathrm{Ct}$ values were used in calculating fold-change according to the $C_{T}$ method [11]. All tumor and normal $C_{T}$ values were first normalized to glyceraldehyde 3-phosphate dehydrogenase $(G A P D H)$. The quantity of genomic material present for each gene in the tumor sample was then normalized to its normal counterpart.

\section{Results}

The patient is a 63-year-old Caucasian man diagnosed with adenocarcinoma of the ampulla of Vater. The patient had a Whipple procedure to resect the head of the pancreas, distal stomach duodenum, distal common bile duct, and gallbladder. The maximum dimension of the tumor, which was present at the junction of the ampullary and duodenal mucosa was $1.5 \mathrm{~cm}$. The tumor invaded into the duodenal muscle wall but no lymphatic or vascular invasion was noted. There was no evidence of neoplasm of the lines of resection and there was no evidence of metastatic carcinoma to the 16 peripancreatic lymph nodes examined microscopically (pathologic TNM (Tumor, Node, Metastasis) stage T2, N0, M0). The patient's past history is significant of having smoked one to two packs per day for 15 years, stopping approximately 16 years before the diagnosis of his adenocarcinoma of the ampulla of Vater.

Massively parallel whole-genome sequencing was performed on genomic DNA from germline and tumor samples using the Life Technologies SOLiD ${ }^{\mathrm{TM}}$ version 4.0 mate-pair chemistry. Basic sequence run statistics based on our analysis pipeline are provided in Table 1. A total of 2.38 and 2.21 billion uniquely mappable reads were generated from germline and tumor DNA, which equates to $108 \mathrm{~Gb}$ and $100 \mathrm{~Gb}$ of uniquely mappable sequence for germline and tumor, respectively. Therefore, we achieved $37 \times$ and $40 \times$ genome coverage for tumor and germline, respectively. We detected a total of 2,771,201 SNPs from the germline genome, $91 \%$ of which are present in $\mathrm{dbSNP}$ (release 129). The transition to transversion ratio was 2.12, which is inline with what would be expected in a diploid human genome [12]. The full genome has been deposited in the database of Genotypes and Phenotypes (dbGaP) of the National Center for Biotechnology Information (submission ID SRA 053213).

To discover somatic mutations within ampullary cancer, we used a custom paired analysis pipeline. The overview of somatic alterations within this tumor is provided in the form of a Circos plot (Figure 1). Our paired analysis revealed 19,143 genome-wide somatic point mutations, of which 30 map within known annotated coding sequences. A list of all somatic missense $(n=28)$ and nonsense mutations $(n=2)$ is provided in Table 2 . The most notable mutation is an activating KRAS (Kirsten rat sarcoma viral oncogene homolog) mutation at codon 12 $(\mathrm{G} 12 \mathrm{~V})$, which is one of the most commonly reported mutations in ampullary carcinomas $[13,14]$. Furthermore, we discovered three somatic small insertions and deletions within coding regions, which result in frameshift mutations (Table 2). All missense mutations were assessed for likely functional consequences using the SIFT prediction algorithm $[15,16]$, which characterized 
Table 1 Basic sequencing statistics

\begin{tabular}{lcccccc}
\hline Genome & $\begin{array}{c}\text { Number of uniquely } \\
\text { mapping 50 bp reads }\end{array}$ & $\begin{array}{c}\text { Number of uniquely } \\
\text { mapping bases }\end{array}$ & $\begin{array}{c}\text { Genome } \\
\text { coverage }\end{array}$ & $\begin{array}{c}\text { Number of } \\
\text { germline variants }\end{array}$ & $\begin{array}{c}\text { Uniquely mapping } \\
\text { tag pairs }\end{array}$ & $\begin{array}{c}\text { Read-pair } \\
\text { coverage }\end{array}$ \\
\hline Germline & $2,383,981,557$ & $108,322,420,859$ & 40 & $2,771,201$ & $887,285,914$ \\
Tumor & $2,215,368,333$ & $100,400,536,852$ & 37 & - & 843 \\
\hline
\end{tabular}

Read pair coverage $=($ Number of uniquely mappable tag-pairs $) \times($ Insert length $) /$ Haploid genome size.

mutations as tolerated or damaging. Of the 28 missense mutations that were assessed, 19 (68\%) were predicted to be damaging. Previously, we calculated the rate of SIFT damaging calls from a random set of approximately 10,000 missense variants from the 1000 Genomes data, which showed a rate of damaging mutations of $15 \%$. Validation by Sanger sequence analysis is presented in Additional file 3.

To identify regions of somatic copy number loss, we utilized a basic algorithm that determined $\log 2$ ratios in

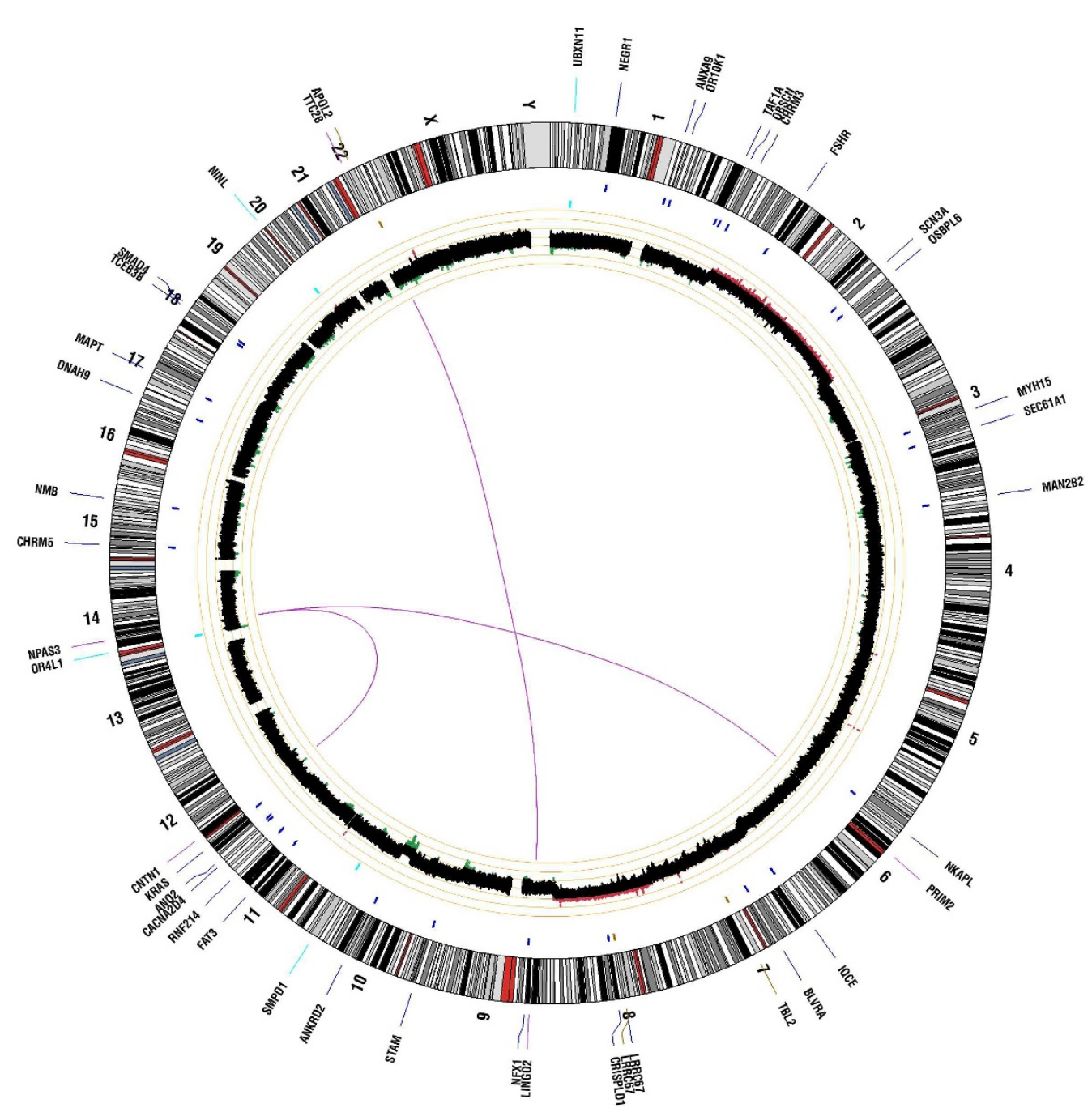

Figure 1 Circos plot summarizing somatic events contained within pancreatic tumor of the ampulla of Vater. The outer ring shows gene symbols for those genes somatically altered in the tumor relative to their map position against the human genome chromosome karyotype. Blue tick marks denote genes containing nonsynonymous point mutations. Cyan tick marks denote genes containing coding indels. Magenta tick marks represent discordant read pairs supporting putative translocation events and those genes involved in breakpoints. The inner ring represents somatic copy number events with regions of gain shown in red and regions of loss shown in green, with brighter colors denoting higher degrees of gain or loss. Magenta lines in the center represent breakpoint regions for translocation events. 
Table 2 List of somatic coding point mutations and small indels

\begin{tabular}{|c|c|c|c|c|}
\hline Genomic position and alleles ${ }^{a}$ & Gene ID & Codon change & Amino acid consequence & SIFT prediction \\
\hline Chr1:71831141G/A & NEGR1 & $503 C>T$ & $\mathrm{~T} 168 \mathrm{I}$ & Damaging \\
\hline Chr1:149227247C/T & ANXA9 & $760 C>T$ & $\mathrm{R} 245 \mathrm{C}$ & Damaging \\
\hline Chr1:156702726C/A & OR10K1 & $751 C>A$ & $\mathrm{H} 251 \mathrm{~N}$ & Damaging \\
\hline Chr1:220801358G/T & TAF1A & $1211 C>A$ & A404D & Damaging \\
\hline Chr1:226621463T/G & OBSCN & $19592 \mathrm{~T}>\mathrm{G}$ & F6531C & Not scored \\
\hline Chr1:238137913G/A & СНRM3 & $539 G>A$ & R180Q & Damaging \\
\hline Chr2:49235000G/A & FSHR & $61 C>T$ & $\mathrm{R} 21 \mathrm{~W}$ & Tolerated \\
\hline Chr2:165735207G/C & SCN3A & $362 C>G$ & A121G & Damaging \\
\hline Chr2:178905886C/T & OSBPL6 & $529 C>T$ & $\mathrm{R} 177 \mathrm{X}$ & - \\
\hline Chr3:109658364C/T & MYH15 & $2137 G>A$ & G713R & Damaging \\
\hline Chr3:129258301T/G & SEC61A1 & $280 \mathrm{~T}>\mathrm{G}$ & L94V & Damaging \\
\hline Chr4:6670038G/A & MAN2B2 & $2732 \mathrm{G}>\mathrm{A}$ & $\mathrm{R} 911 \mathrm{H}$ & Tolerated \\
\hline Chr6:28336272A/G & NKAPL & $1144 A>G$ & S382G & Damaging \\
\hline Chr7:2599270C/T & IQCE & $1333 C>T$ & R445X & - \\
\hline Chr7:43813258G/A & BLVRA & $790 G>A$ & G264S & Tolerated \\
\hline Chr8:76091857G/T & CRISPLD1 & $950 \mathrm{G}>\mathrm{T}$ & C317F & Damaging \\
\hline Chr9:33301117G/A & NFX1 & $1240 G>A$ & G414R & Damaging \\
\hline Chr10:17777173T/C & STAM & $364 T>C$ & $\mathrm{Y} 122 \mathrm{H}$ & Damaging \\
\hline Chr10:99328071C/T & ANKRD2 & $274 C>T$ & R92W & Damaging \\
\hline Chr11:6368506-6368518del & SMPD1 & 102-114del & L35WfsX72 & - \\
\hline Chr11:92170718A/T & FAT3 & $4894 A>T$ & M1632L & Not scored \\
\hline Chr11:116661033A/T & RNF214 & $2068 \mathrm{~A}>\mathrm{T}$ & T690S & Not scored \\
\hline Chr12:1854754C/T & CACNA2D4 & $1730 G>A$ & G577E & Damaging \\
\hline Chr12:5902149C/A & ANO2 & $93 \mathrm{G}>\mathrm{T}$ & Q31H & Damaging \\
\hline Chr12:25289551C/A & KRAS & $35 G>T$ & G12V & Damaging \\
\hline Chr14:19598288-19598307del & ORALI & 245-264del & $182 T f S X 104$ & - \\
\hline Chr15:32142462G/A & CHRM5 & $252 \mathrm{G}>\mathrm{A}$ & M84l & Damaging \\
\hline Chr15:83002258G/C & $N M B$ & $130 C>G$ & $\mathrm{H} 44 \mathrm{D}$ & Tolerated \\
\hline Chr17:11543819G/A & DNAH9 & $4919 G>A$ & R1640Q & Tolerated \\
\hline Chr17:41416912C/G & MAPT & $905 C>G$ & $\mathrm{~T} 302 \mathrm{R}$ & Damaging \\
\hline Chr18:42815392C/T & TCEB3B & $242 \mathrm{G}>\mathrm{A}$ & R81Q & Tolerated \\
\hline Chr18:46845916C/T & SMAD4 & $1081 C>T$ & R361C & Damaging \\
\hline Chr20:25405049-25405055del & NINL & 2872-2878del & W958HfsX960 & - \\
\hline
\end{tabular}

${ }^{\mathrm{a}}$ Reference allele/mutated allele.

coverage difference between tumor and germline over a sliding window of $4,000 \mathrm{bp}$. Regions of copy number gain or loss are shown in Figure 1. This tumor exhibited whole chromosome copy number gains of chromosomes 2 and 8 , along with copy number loss of chromosome 19 . Of most significance was an approximate $20 \mathrm{Mb}$ interstitial deletion at $10 \mathrm{q} 23$, which also contained a more focal region $(2 \mathrm{Mb})$ of homozygous loss that encompassed the PTEN tumor suppressor gene (Figure 2). No other regions of focal gain or amplification were detected in this tumor (validation data are presented in Additional file 4).

To identify potential cis chromosomal rearrangements and translocation events, we searched for significant evidence of discordant mate pairs. The long insert mate pairs provide improved power for detecting structural alterations through improved clonal coverage. Clonal coverage can be defined as the genomic coverage (that is, $30 \times)$ multiplied by the length of the insert $(1,500 \mathrm{bp})$, divided by the amount of sequence derived from each mate pair (100 bp). For example, at $37 \times$ genomic coverage for our tumor specimen and with 1,500 bp average matepair insert size, and with $2 \times 50$ bp mate-pairs (or $100 \mathrm{bp}$ total), we achieve a clonal coverage of $432 \times$. With such high clonal coverage we have significant power to detect evidence of discordant mate-pair reads, where the length of the insert deviates substantially from the mean insert length and/or map to different chromosomes or chromosomal regions. Utilizing an algorithm that identified discordant mate-pairs specific to the tumor, we discovered two independent translocation events occurring in the tumor. Both events involve genes on each side of the translocation event. One event is evidenced by significant 


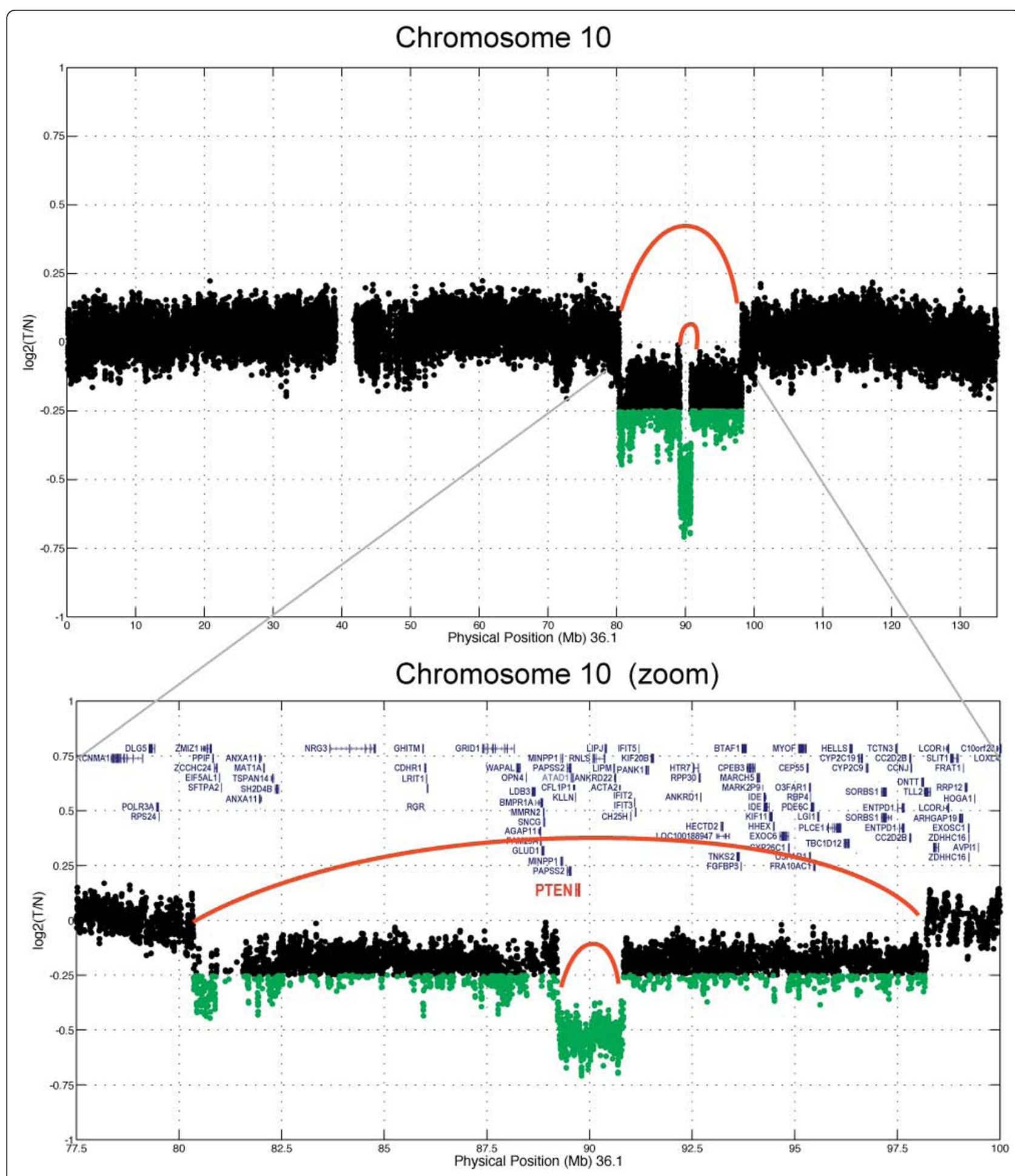

Figure 2 Zoom in of the $10 \mathrm{q}$ region containing focal homozygous deletion encompassing the PTEN tumor suppressor gene.

discordant read pairs in the tumor mapping to the LINGO2 (leucine rich repeat and Ig domain containing 2) locus at 9q21.1 (chr9: 27990017-27991975), which is translocated to the TTC28 (tetratricopeptide repeat domain 28) locus at 22q12.1 (chr22: 27401302-27401562) (Figure 1). A second event is evidenced by discordant mate-pair read mapping to the PRIM2 (primase, DNA, polypeptide 2) locus at 6p12.1 (chr6: 57450028- 57451992) and to the 
NPAS3 (neuronal PAS domain protein 3) locus at 14q13.1 (chr14: 33206124- 33207653) (Figure 1).

\section{Discussion}

Adenocarcinomas of the ampulla of Vater are relatively rare, accounting for only $0.2 \%$ of gastrointestinal cancers [17]. Perhaps due to their location and propensity to present with jaundice at an early resectable stage, these tumors are more likely to be resectable at the time of diagnosis than are pancreatic cancers [18]. Furthermore, in comparison to pancreatic cancer, resected ampullary cancers are associated with better 5-year survival rates of 34 to $61 \%$ [19-21]. Surgical series have demonstrated the factors affecting survival include completeness of surgical resection and nodal status. Surgical treatment for ampullary cancer and cancers in the head of the pancreas are similar in that surgeons perform a pancreaticoduodenectomy. Thereafter, the treatments may diverge. There is no clear consensus on the role of or the optimal regimen for adjuvant chemotherapy in ampullary cancers. Similarly, in part due to its relative rarity, there is no clear standard chemotherapeutic regimen for recurrent or metastatic ampullary cancer.

A better understanding of molecular oncogenesis and the emergence of targeted agents will likely lead to improved treatment outcomes in this and other cancers. Our study used whole genome sequencing to analyze the genome of a resected ampullary carcinoma. We found expected as well as novel aberrations. We found an activating mutation in KRAS codon 12. KRAS mutations are common in ampullary cancer although the 25 to $37 \%$ incidence appears to be lower than the approximately 95\% rate of KRAS mutation seen in pancreatic adenocarcinomas $[13,14,22,23]$. Furthermore, similar to what is seen in colonic adenomas, KRAS mutations occur in benign ampullary adenomas, suggesting activating mutations of KRAS are relatively early events in the progression toward cancer and the mutation does not appear to affect prognosis [14]. This tumor also demonstrated a somatic nonsynonymous mutation in SMAD4 (mothers against decapentaplegic homolog 4), which has been observed previously in $50 \%$ of ampullary cancers but infrequently in bile duct cancers [24].

The most notable gene deletion we found was a focal deletion of a region in chromosome 10 including the PTEN tumor suppressor gene (phosphate and tensin homologue deleted on chromosome 10). Cowden's syndrome is characterized by a germline mutation in the PTEN gene resulting in loss of function. This syndrome is characterized by noncancerous hamartomas of the skin and mucous membranes and affected patients have in increased risk of tumors of the breast, thyroid, uterus and gastrointestinal tract. Benign tumors of the ampulla of Vater have been reported in patients with Cowden's syndrome but are not a common feature within cancers of the ampulla. Loss of PTEN expression by immunohistochemisty has been associated with liver metastases and poor prognosis in colon cancer [25]. In a large-scale survey of the genomic aberrations of pancreatic cancers, PTEN deletions were not seen, although small deleterious coding mutations were detected [26]. We can conclude that despite their anatomic location in proximity to the pancreas, ampullary cancers are distinct entities from adenocarcinoma of the pancreas and bile duct cancers and thus should be treated as a different entity.

To that end, the loss of PTEN expression is important not only in the pathogenesis but because it exposes a potential therapeutic target (Figure 3). The PTEN protein product is an inhibitor of phosphoinositide 3-kinase (PI3K) and downstream signaling through AKT. Phosphorylation of Akt results in phosphorylation of several target proteins involved in regulation of key cellular functions, including cell proliferation, glucose metabolism, protein translation, and cell survival [27]. Additionally, activation of the PI3K pathway has been linked to activation of mammalian target of rapamycin (mTOR), although the mechanism is not yet fully elucidated [28]. The presence of a deletion in PTEN in this ampullary cancer would be predicted to release from inhibition activation of the PI3K/mTOR pathway. Consequently, one can infer that an agent that is a dual PI3K/mTOR inhibitor, such as NVP-BEZ235, would be an attractive therapeutic option for our patient should his disease recur [29]. NVP-BEZ235 and other agents like it have been shown in vitro to inhibit growth of cancer cells with activating mutations of PI3K and are all under clinical development [30]. In the case presented here, however, the tumor carries both a KRAS activating mutation and complete inactivation of PTEN, supporting dual activation of both the MEK/ERK and the PI3K/AKT axes (Figure 3). The inhibition of only one axis may not be sufficient for effective treatment as there is likely to be compensatory activity from the other activated axis.

Our group reported the beneficial results seen in a clinical trial on patients with refractory solid tumors whose chemotherapy was chosen based on analysis of tumor biopsies using immunohistochemistry and expression arrays [31]. New technologies such as applied herein have made high-throughput whole-genome sequencing a more rapid and cost-effective process in a manner not possible with older technologies such as Sanger sequencing. The prospect is raised, therefore, that one may soon be able to apply whole-genome sequencing to the analysis of an individual patient's tumor to guide an informed choice of a therapeutic regimen. This type of personalized or precision medicine has only begun to be studied. Several limitations remain before this whole-genome sequencing methodology can be widely applied, including the need for improved and standardized bioinformatic 


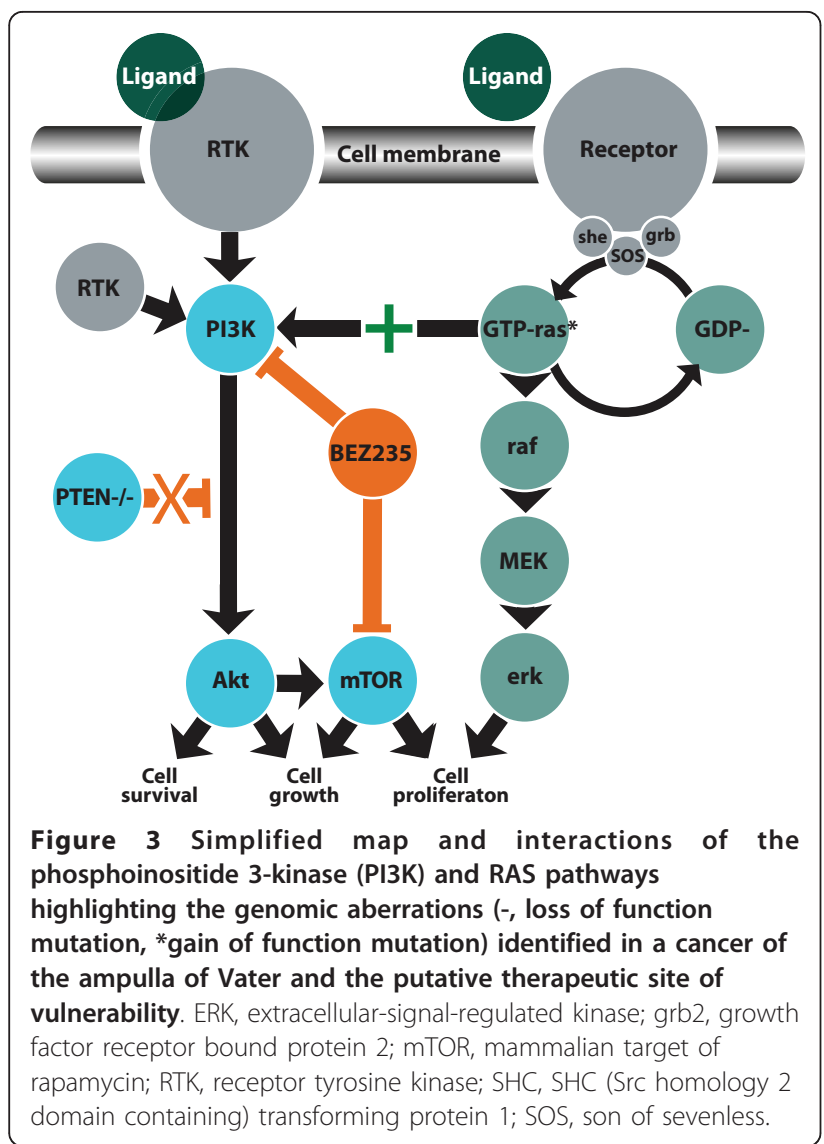

analysis, along with reliable and rapid methods for validation of genomic findings and cost. Furthermore, if a target is found, one must have access to an agent and, in many cases, such agents may not be approved for clinical use. Thus, we must begin to understand the links between genomic profile and drug context in early drug development. This is amplified even more where there is evidence to support combination therapies.

\section{Conclusions}

We have analyzed the whole genome sequence of a cancer of the ampulla of Vater to uncover the compendium of somatic events occurring in this tumor. Among the mutations discovered were those that might be considered potential therapeutic vulnerabilities. As whole-genome sequencing becomes more rapid and less expensive, the potential for targeted and truly personalized treatments increases. Consequently, as we continue to refine our abilities to uncover the full landscape of somatic alterations, we must in parallel continue innovative drug development methods, including preclinical and early phase I combination trials. This will allow us to understand toxicities and appropriate dosing regimens, to obtain the safest and most appropriate combinations matched to specific genomic and molecular contexts.

\section{Additional material}

Additional file 1: Supplemental methods giving additional details regarding the methods used for the detection of somatic translocations and intrachromosomal rearrangements. Additional file 2: Validation primer sets: sequencing and quantitative PCR primer pairs used in validation of next generation sequencing results.

Additional file 3: Figure showing the results of validation by Sanger sequencing: validation of single nucleotide variants $(n=10)$ and a local deletion. Sequencing electropherograms depicting specific mutations in selected genes in forward and reverse orientations.

Additional file 4: Figure demonstrating the validation of the next generation sequencing comparative genomic hybridization findings of a PTEN deletion. Results depicted show the relative fold changes measured by quantitative PCR in tumor versus normal genomic DNA in specific regions of chromosome $10 \mathrm{q}$ affected by hemi- (RGR and HHEX) or homozygous (PTEN) deletion.

\section{Abbreviations}

BAM: binary sequence alignment map; bp: base pair; mTOR: mammalian target of rapamycin; PCR: polymerase chain reaction; PI3K: phosphoinositide 3-kinase; SNP: single nucleotide polymorphism; WIRB: Western Institutional Review Board.

\section{Acknowledgements}

Support for this project was provided by National Cancer Institute P01 Grant CA109552. This article is funded in part by the Life Technologies ${ }^{\mathrm{TM}}$ Foundation. Authors GH, JMT and DDVH are supported by a Stand Up To Cancer Dream Team Translational Cancer Research Grant, a Program of the Entertainment Industry Foundation (SU2C-AACR-DT0509).

\section{Author details}

${ }^{1}$ Integrated Cancer Genomics Division, Translational Genomics Research Institute, 445 N. Fifth Ave, Phoenix, AZ 85004, USA. ${ }^{2}$ Virginia G Piper Cancer Center, 10290 N. 92nd St, Scottsdale, AZ 85258, USA. ${ }^{3}$ Neurogenomics Division, Translational Genomics Research Institute, 445 N. Fifth Ave, Phoenix, AZ 85004, USA. ${ }^{4}$ Clinical Translational Research Division, Translational Genomics Research Institute, 445 N. Fifth Ave, Phoenix, AZ 85004, USA. ${ }^{5}$ Department of Surgery, Banner Good Samaritan Medical Center, 1111 E. McDowell Rd, Phoenix, AZ 85006, USA. ${ }^{6}$ School of Life Sciences, Arizona State University, 427 E. Tyler Mall MC 5401 SOLS, Phoenix, AZ 85287, USA.

\section{Authors' contributions}

MJD, DWC, DVH, and JC conceived of the study, and participated in its design and coordination, and drafted the manuscript. LK and JT participated in study design and coordination. SS participated in the sequence alignment and manuscript drafting. TMM, JD, and IC carried out molecular genetic and sequencing studies. $A C, T I$, and $J A$ participated in sequence alignment. $A B$, $A W, A D, D L, G H$ and LK participated in sample acquisition, sample quality assessment, and preparation. All authors read and approved the final manuscript.

\section{Competing interests}

The authors declare that they have no competing interests.

Received: 6 September 2011 Revised: 29 May 2012

Accepted: 4 July 2012 Published: 4 July 2012

\section{References}

1. Valle J, Wasan H, Palmer DH, Cunningham D, Anthoney A, Maraveyas A, Madhusudan S, Iveson T, Hughes S, Pereira SP, Roughton M, Bridgewater J: Cisplatin plus gemcitabine versus gemcitabine for biliary tract cancer. $N$ Engl J Med 2010, 362:1273-1281.

2. Druker BJ, Guilhot F, O'Brien SG, Gathmann I, Kantarjian H, Gattermann N, Deininger MW, Silver RT, Goldman JM, Stone RM, Cervantes F, Hochhaus A, Powell BL, Gabrilove JL, Rousselot P, Reiffers J, Cornelissen JJ, Hughes T, 
Agis H, Fischer T, Verhoef G, Shepherd J, Saglio G, Gratwohl A, Nielsen JL, Radich JP, Simonsson B, Taylor K, Baccarani M, So C, et al: Five-year followup of patients receiving imatinib for chronic myeloid leukemia. $N$ Engl J Med 2006, 355:2408-2417.

3. Blanke $C D$, Demetri $G D$, von Mehren $M$, Heinrich $M C$, Eisenberg $B$ Fletcher JA, Corless CL, Fletcher CD, Roberts PJ, Heinz D, Wehre E, Nikolova Z, Joensuu H: Long-term results from a randomized phase II trial of standard- versus higher-dose imatinib mesylate for patients with unresectable or metastatic gastrointestinal stromal tumors expressing KIT. J Clin Oncol 2008, 26:620-625.

4. Von Hoff DD, LoRusso PM, Rudin CM, Reddy JC, Yauch RL, Tibes R, Weiss GJ, Borad MJ, Hann CL, Brahmer JR, Mackey HM, Lum BL, Darbonne WC, Marsters JC Jr, de Sauvage FJ, Low JA: Inhibition of the hedgehog pathway in advanced basal-cell carcinoma. N Engl J Med 2009, 361:1164-1172.

5. Flaherty KT, Puzanov I, Kim KB, Ribas A, McArthur GA, Sosman JA, O'Dwyer PJ, Lee RJ, Grippo JF, Nolop K, Chapman PB: Inhibition of mutated, activated BRAF in metastatic melanoma. N Engl J Med 2010, 363:809-819.

6. Tuch BB, Laborde RR, Xu X, Gu J, Chung CB, Monighetti CK, Stanley SJ, Olsen KD, Kasperbauer JL, Moore EJ, Broomer AJ, Tan R, Brzoska PM, Muller MW, Siddiqui AS, Asmann YW, Sun Y, Kuersten S, Barker MA, De La Vega FM, Smith DI: Tumor transcriptome sequencing reveals allelic expression imbalances associated with copy number alterations. PLoS One 2010, 5:e9317.

7. Robbins CM, Tembe WA, Baker A, Sinari S, Moses TY, BeckstromSternberg S, Beckstrom-Sternberg J, Barrett M, Long J, Chinnaiyan A, Lowey J, Suh E, Pearson JV, Craig DW, Agus DB, Pienta KJ, Carpten JD: Copy number and targeted mutational analysis reveals novel somatic events in metastatic prostate tumors. Genome Res 2011, 21:47-55.

8. McKenna A, Hanna M, Banks E, Sivachenko A, Cibulskis K, Kernytsky A, Garimella K, Altshuler D, Gabriel S, Daly M, DePristo MA: The Genome Analysis Toolkit: a MapReduce framework for analyzing next-generation DNA sequencing data. Genome Res 2010, 20:1297-1303.

9. Stajich JE, Block D, Boulez K, Brenner SE, Chervitz SA, Dagdigian C Fuellen G, Gilbert JG, Korf I, Lapp H, Lehvaslaiho H, Matsalla C, Mungall CJ, Osborne Bl, Pocock MR, Schattner P, Senger M, Stein LD, Stupka E, Wilkinson MD, Birney E: The Bioperl toolkit: Perl modules for the life sciences. Genome Res 2002, 12:1611-1618.

10. Li H, Handsaker B, Wysoker A, Fennell T, Ruan J, Homer N, Marth G, Abecasis G, Durbin R: The Sequence Alignment/Map format and SAMtools. Bioinformatics 2009, 25:2078-2079.

11. Schmittgen TD, Livak KJ: Analyzing real-time PCR data by the comparative C(T) method. Nat Protoc 2008, 3:1101-1108.

12. A map of human genome variation from population-scale sequencing. Nature 2010, 467:1061-1073.

13. Scarpa A, Zamboni G, Achille A, Capelli P, Bogina G, lacono C, Serio G, Accolla RS: ras-family gene mutations in neoplasia of the ampulla of Vater. Int J Cancer 1994, 59:39-42.

14. Howe JR, Klimstra DS, Cordon-Cardo C, Paty PB, Park PY, Brennan MF: K-ras mutation in adenomas and carcinomas of the ampulla of vater. Clin Cancer Res 1997, 3:129-133.

15. Ng PC, Henikoff S: Predicting deleterious amino acid substitutions. Genome Res 2001, 11:863-874.

16. Ng PC, Henikoff S: SIFT: Predicting amino acid changes that affect protein function. Nucleic Acids Res 2003, 31:3812-3814.

17. Howe JR, Klimstra DS, Moccia RD, Conlon KC, Brennan MF: Factors predictive of survival in ampullary carcinoma. Ann Surg 1998, 228:87-94.

18. Yeo CJ, Sohn TA, Cameron JL, Hruban RH, Lillemoe KD, Pitt HA: Periampullary adenocarcinoma: analysis of 5-year survivors. Ann Surg 1998, 227:821-831.

19. Monson JR, Donohue JH, McEntee GP, Mcllrath DC, van Heerden JA, Shorter RG, Nagorney DM, Ilstrup DM: Radical resection for carcinoma of the ampulla of Vater. Arch Surg 1991, 126:353-357.

20. O'Connell JB, Maggard MA, Manunga J Jr, Tomlinson JS, Reber HA, Ko CY, Hines OJ: Survival after resection of ampullary carcinoma: a national population-based study. Ann Surg Oncol 2008, 15:1820-1827.

21. Shutze WP, Sack J, Aldrete JS: Long-term follow-up of 24 patients undergoing radical resection for ampullary carcinoma, 1953 to 1988. Cancer 1990, 66:1717-1720
22. Rozenblum E, Schutte M, Goggins M, Hahn SA, Panzer S, Zahurak M, Goodman SN, Sohn TA, Hruban RH, Yeo CJ, Kern SE: Tumor-suppressive pathways in pancreatic carcinoma. Cancer Res 1997, 57:1731-1734.

23. Demeure MJ, Doffek KM, Komorowski RA, Wilson SD: Adenocarcinoma of the pancreas: detection of occult metastases in regional lymph nodes by a polymerase chain reaction-based assay. Cancer 1998, 83:1328-1334.

24. Suto T, Sugai T, Habano W, Uesugi N, Kanno S, Saito K, Nakamura S: Allelotype analysis of the PTEN, Smad4 and DCC genes in biliary tract cancer. Anticancer Res 2002, 22:1529-1536.

25. Sawai H, Yasuda A, Ochi N, Ma J, Matsuo Y, Wakasugi T, Takahashi H, Funahashi H, Sato M, Takeyama H: Loss of PTEN expression is associated with colorectal cancer liver metastasis and poor patient survival. BMC Gastroenterol 2008, 8:56.

26. Jones $S$, Zhang $X$, Parsons DW, Lin JC, Leary RJ, Angenendt $P$, Mankoo $P$, Carter H, Kamiyama H, Jimeno A, Hong SM, Fu B, Lin MT, Calhoun ES, Kamiyama M, Walter K, Nikolskaya T, Nikolsky Y, Hartigan J, Smith DR, Hidalgo M, Leach SD, Klein AP, Jaffee EM, Goggins M, Maitra A, lacobuzioDonahue C, Eshleman JR, Kern SE, Hruban RH, et al: Core signaling pathways in human pancreatic cancers revealed by global genomic analyses. Science 2008, 321:1801-1806.

27. Paez J, Sellers WR: PI3K/PTEN/AKT pathway. A critical mediator of oncogenic signaling. Cancer Treat Res 2003, 115:145-167.

28. Gingras $A C$, Raught $B$, Sonenberg N: Regulation of translation initiation by FRAP/mTOR. Genes Dev 2001, 15:807-826.

29. Maira SM, Stauffer F, Brueggen J, Furet P, Schnell C, Fritsch C, Brachmann S, Chene P, De Pover A, Schoemaker K, Fabbro D, Gabriel D, Simonen M, Murphy L, Finan P, Sellers W, Garcia-Echeverria C: Identification and characterization of NVP-BEZ235, a new orally available dual phosphatidylinositol 3-kinase/mammalian target of rapamycin inhibitor with potent in vivo antitumor activity. Mol Cancer Ther 2008, 7:1851-1863.

30. Serra V, Markman B, Scaltriti M, Eichhorn PJ, Valero V, Guzman M, Botero ML, Llonch E, Atzori F, Di Cosimo S, Maira M, Garcia-Echeverria C, Parra JL, Arribas J, Baselga J: NVP-BEZ235, a dual PI3K/mTOR inhibitor, prevents PI3K signaling and inhibits the growth of cancer cells with activating PI3K mutations. Cancer Res 2008, 68:8022-8030.

31. Von Hoff DD, Stephenson JJ Jr, Rosen P, Loesch DM, Borad MJ, Anthony S, Jameson G, Brown S, Cantafio N, Richards DA, Fitch TR, Wasserman E, Fernandez C, Green S, Sutherland W, Bittner M, Alarcon A, Mallery D, Penny R: Pilot study using molecular profiling of patients' tumors to find potential targets and select treatments for their refractory cancers. J Clin Oncol 2010, 28:4877-4883.

\section{doi:10.1186/gm357}

Cite this article as: Demeure et al:: Cancer of the ampulla of Vater: analysis of the whole genome sequence exposes a potential therapeutic vulnerability. Genome Medicine 2012 4:56.

\section{Submit your next manuscript to BioMed Central and take full advantage of:}

- Convenient online submission

- Thorough peer review

- No space constraints or color figure charges

- Immediate publication on acceptance

- Inclusion in PubMed, CAS, Scopus and Google Scholar

- Research which is freely available for redistribution

Submit your manuscript at www.biomedcentral.com/submit
Ciomed Central 